\title{
Switching Gain Reduction in Adaptive Sliding Mode Control for Rigid Spacecraft Attitude Maneuvers
}

\author{
Zhen Chen, ${ }^{1,2}$ Binglong Cong, ${ }^{1,2}$ and Xiangdong Liu ${ }^{1,2}$ \\ ${ }^{1}$ School of Automation, Beijing Institute of Technology, Beijing 100081, China \\ ${ }^{2}$ Key Laboratory for Intelligent Control \& Decision of Complex Systems, Beijing Institute of Technology, Beijing 100081, China \\ Correspondence should be addressed to Binglong Cong; cbl@bit.edu.cn
}

Received 15 December 2012; Revised 20 March 2013; Accepted 27 March 2013

Academic Editor: Jui-Sheng Lin

Copyright (c) 2013 Zhen Chen et al. This is an open access article distributed under the Creative Commons Attribution License, which permits unrestricted use, distribution, and reproduction in any medium, provided the original work is properly cited.

\begin{abstract}
This paper investigates the overadaptation problem in current adaptive sliding mode control (ASMC) for rigid spacecraft attitude maneuvers. The inertia matrix uncertainty and external disturbance are taken into account, and an adaptive scheme is employed for the switching gain calculation. A detailed analysis of existing ASMC design reveals the fact that the switching gain would be overestimated if the ASMC algorithm is developed in the framework of conventional sliding mode control (SMC), owing to the unrelated adaptation caused by initial tracking error. The global sliding mode concept of integral sliding mode control (ISMC) is exploited to solve such a problem. The advantages of the proposed strategy are twofold. First, a much smaller switching gain is generated as compared to conventional ASMC. Second, the resulting small switching gain would not slow down the system response. The advantages of the proposed strategy are verified by both theoretical analysis and simulation results.
\end{abstract}

\section{Introduction}

Considering the facts that the governing equations for attitude motion are highly nonlinear and the spacecraft commonly operates in the presence of various external disturbances and parametric uncertainties, robust nonlinear control approaches are preferred over linear control methods to achieve the desired performance. Among prevalent nonlinear control techniques, sliding mode control (SMC) is recognized as a powerful but easy-to-implement design tool for uncertain nonlinear systems, owing to its excellent properties such as disturbance rejection, insensitivity to parameter variation, and finite-time response. Applying SMC to spacecraft attitude regulation and tracking problems has been thoroughly studied in the past two decades [1-14].

In order to obtain attitude controllers via SMC, two major parameters should be determined, that is, the sliding function gain and the switching gain. On the one hand, the sliding function gain is directly related to the sliding function dynamics, where all the specifications to achieve the required system performance should be taken into account. To obtain the SMC algorithm for attitude regulation, an optimal control problem was solved to synthesize the sliding function gain in [1], where a quadratic performance index constructed by quaternion and angular velocity was minimized. Such an optimal sliding function was described as a linear function of attitude parameter and angular velocity. Until now, most SMC algorithms for attitude control are still based on such a kind of linear sliding function for its optimization and easy implementation, as suggested in [2-9]. However, as discussed in [10], independent response for each attitude parameter component could not be realized by those linear sliding functions for the failure in accounting for the nonlinearity in attitude kinematics. Hence, nonlinear sliding functions exploiting the characteristics in attitude kinematics are studied to achieve a decoupled attitude parameter response; see, for example [10-12].

On the other hand, the switching gain selection is a challenging issue. In the sense of guaranteeing the system stability, the switching gain should be chosen at least larger than the upper bound on the lumped uncertainty (the combined effect caused by parametric uncertainty and external disturbance) to satisfy the so-called reaching condition. Hence, most SMCbased attitude control laws assume that the upper bound is available in advance for the switching gain tuning $[1-8,10-$ 14]. Nonetheless, in many practical situations, such an upper 
bound may not be easily obtained due to the complexity and unpredictability of the lumped uncertainty. As a result, techniques removing the requirement of a prior knowledge of the upper bound are investigated. One way is using the disturbance observer (DOB) approach, as suggested in [1517]. In [15], the DOB was utilized to improve the control accuracy when the boundary layer technique was employed to soften the chattering. In [16], the lumped uncertainty was assumed to be generated by a linear exogenous system, and a DOB was then designed. However, such an assumption may be hard to satisfy. Moreover, as pointed out in [17], one possible problem in those DOB-based SMC algorithms is the system stability analysis under composite controller, and also a conservative switching gain is needed to complete the controller design. The other effective approach is integrating adaptive strategy into SMC design, known as the adaptive sliding mode control (ASMC) technique in the literature [1822]. The main idea of ASMC design lies in the fact that the switching gain can be adjusted by the departure from the sliding surface, and the upper bound on the lumped uncertainty is not required to be known in advance. Compared to the DOB-based SMC design, ASMC is simple to implement and involves straightforward tuning, which has been successfully applied to the attitude control problem $[9,23,24]$.

One problem in ASMC technique is the parameter drift phenomenon, where the switching gain will become unbounded if the sliding function is not identically equal to zero in practical applications. Fortunately, with the development of adaptive control theory, various solutions have been proposed to solve such a problem, such as the $\sigma$ modification, $e$-modification, and the dead zone method [25], which can be directly applied to the ASMC design. However, there is another problem in existing ASMC design which has not been fully addressed, that is, the overadaptation problem, where the switching gain is unnecessarily large with respect to the actual bound on the lumped uncertainty even if no parameter drift exists. As the chattering level is proportional to the switching gain in discontinuous ASMC laws, the overadaptation problem will lead to a serious chattering phenomenon. While for the ASMC algorithms using continuous approximation techniques such as the boundary layer method, an overestimated switching gain may induce undesired large-amplitude jump in control signal.

In this paper, we aim to address the overadaptation problem by focusing on the attitude tracking control problem of a rigid spacecraft involving inertia matrix uncertainty and external disturbance. However, the proposed strategy can be easily extended to the more general nonlinear systems with bounded uncertain parameters as studied in [22]. The main contribution of this paper is that the switching gain generated by the proposed ASMC algorithm is more close to the actual one. Hence, a lower-chattering control signal will be produced by the discontinuous ASMC law, and the large-amplitude control jump will not exist for the continuous counterpart. The reminder of this paper is organized as follows. Section 2 states the attitude tracking control problem and introduces some preliminaries about current ASMC design. Section 3 analyzes the underlying reason for the overadaptation problem and presents the main result. Section 4 verifies the validity of the proposed strategies by comparing with current ASMC algorithm in a numerical simulation. Section 5 concludes this paper.

\section{Preliminaries}

2.1. Problem Statement. Three coordinate frames will be used in this paper, which are the body fixed frame $\mathscr{F}_{B}$, the inertial reference frame $\mathscr{F}_{I}$, and the desired frames $\mathscr{F}_{D}$. Unless otherwise specified, all the vector quantities are expressed in their corresponding frames, which accords with practical situations. It is assumed that the attitude parameter and the angular velocity measurements are available in the feedback controller design and the actuator dynamics is neglected.

Consider a thruster-controlled rigid spacecraft whose attitude dynamics and kinematics are described by the following equations [11]:

$$
\begin{gathered}
J \dot{\omega}+\omega^{\times} J \omega=u+d, \\
\dot{\sigma}=M(\sigma) \omega,
\end{gathered}
$$

where $J \in \mathbb{R}^{3 \times 3}$ is the inertia matrix of the spacecraft, $\omega \in$ $\mathbb{R}^{3}$ is the angular velocity of $\mathscr{F}_{B}$ with respect to $\mathscr{F}_{I}$, the superscript $(\cdot)^{\times}$is the skew-symmetric matrix operator on any vector $\alpha=\left[\alpha_{1}, \alpha_{2}, \alpha_{3}\right]^{T} \in \mathbb{R}^{3}$ such that

$$
\alpha^{\times}=\left[\begin{array}{ccc}
0 & -\alpha_{3} & \alpha_{2} \\
\alpha_{3} & 0 & -\alpha_{1} \\
-\alpha_{2} & \alpha_{1} & 0
\end{array}\right]
$$

$u \in \mathbb{R}^{3}$ and $d \in \mathbb{R}^{3}$ are the vectors of control torque provided by the thrusters and external disturbance, respectively. $\sigma \in$ $\mathbb{R}^{3}$ denotes the modified Rodrigues parameter (MRP) representation for the attitude of $\mathscr{F}_{B}$ with respect to $\mathscr{F}_{I}$. And $M$ : $\mathbb{R}^{3} \rightarrow \mathbb{R}^{3 \times 3}$ is the corresponding Jacobian matrix such that

$$
M(\sigma)=\frac{\left(1-\|\sigma\|^{2}\right) I_{3}+2 \sigma^{\times}+2 \sigma \sigma^{T}}{4},
$$

where $I_{3}$ is the $3 \times 3$ identity matrix and $\|\cdot\|$ is the vector 2-norm. Moreover, $M^{-1}=M^{T}(\sigma) / m(\sigma)$ with $m(\sigma)=\left(1+\|\sigma\|^{2}\right)^{2} / 16$.

With respect to attitude tracking, denote the attitude variables of $\mathscr{F}_{D}$ with respect to $\mathscr{F}_{I}$ as $\sigma_{d} \in \mathbb{R}^{3}$ and $\omega_{d} \in \mathbb{R}^{3}$. Then, the MRP error, $\sigma_{e} \in \mathbb{R}^{3}$, is defined as

$$
\sigma_{e}=\sigma \oplus \sigma_{d}^{*}
$$

Here, $\oplus$ is the MRP product operator, characterizing the successive rotations. For two MRPs expressed in their corresponding frames, for example, $\sigma_{1}$ and $\sigma_{2}$, it is calculated as follows:

$$
\sigma_{1} \oplus \sigma_{2}=\frac{\left(1-\left\|\sigma_{2}\right\|^{2}\right) \sigma_{1}+\left(1-\left\|\sigma_{1}\right\|^{2}\right) \sigma_{2}-2 \sigma_{1}^{\times} \sigma_{2}}{1+\left\|\sigma_{1}\right\|^{2}\left\|\sigma_{2}\right\|^{2}-2 \sigma_{1}^{T} \sigma_{2}}
$$

And the superscript $(\cdot)^{*}$ denotes the complex conjugate of MRP and $\sigma_{d}^{*}=-\sigma_{d}$. 
Furthermore, the angular velocity error, $\omega_{e} \in \mathbb{R}^{3}$, is defined as

$$
\omega_{e}=\omega-R\left(\sigma_{e}\right) \omega_{d}
$$

where $R(\cdot): \mathbb{R}^{3} \rightarrow \mathbb{R}^{3 \times 3}$ is the rotation matrix. Take $\sigma_{e}$ for example, one has

$$
R\left(\sigma_{e}\right)=I_{3}+\frac{8 \sigma_{e}^{\times} \sigma_{e}^{\times}-4\left(1-\left\|\sigma_{e}\right\|^{2}\right) \sigma_{e}^{\times}}{\left(1+\left\|\sigma_{e}\right\|^{2}\right)^{2}} .
$$

Then, the dynamics and kinematics in terms of attitude error variables are described as

$$
\begin{gathered}
J \dot{\omega}_{e}=-\left[\omega_{e}+R\left(\sigma_{e}\right) \omega_{d}\right]^{\times} J\left[\omega_{e}+R\left(\sigma_{e}\right) \omega_{d}\right] \\
+J \omega_{e}^{\times} R\left(\sigma_{e}\right) \omega_{d}-J R\left(\sigma_{e}\right) \dot{\omega}_{d}+u+d, \\
\dot{\sigma}_{e}=M\left(\sigma_{e}\right) \omega_{e} .
\end{gathered}
$$

For clarity, the related arguments will be ignored in the following content, for example, $M\left(\sigma_{e}\right), m\left(\sigma_{e}\right)$, and $R\left(\sigma_{e}\right)$ will be denoted by $M, m$, and $R$, respectively. Furthermore, the uncertainty on the inertia matrix is taken into account, where $J=\widehat{J}+\Delta J$ with $\widehat{J}=\operatorname{diag}\left(\widehat{J}_{1}, \widehat{J}_{2}, \widehat{J}_{3}\right)$ the nominal inertia matrix and $\Delta J$ the uncertainty caused by the changing in mass properties. Then, (9) is rewritten as

$$
\begin{gathered}
\widehat{J} \dot{\omega}_{e}=-\left(\omega_{e}+R \omega_{d}\right)^{\times} \widehat{J}\left(\omega_{e}+R \omega_{d}\right) \\
+\widehat{J} \omega_{e}^{\times} R \omega_{d}-\widehat{J} R \dot{\omega}_{d}+u+u_{d}, \\
\dot{\sigma}_{e}=M \omega_{e},
\end{gathered}
$$

where $u_{d}=d-\left(\omega_{e}+R \omega_{d}\right)^{\times} \Delta J\left(\omega_{e}+R \omega_{d}\right)+\Delta J \omega_{e}^{\times} R \omega_{d}-$ $\Delta J R \dot{\omega}_{d} \in \mathbb{R}^{3}$ stands for the lumped uncertainty. Without loss of generality, it is assumed that $\left\|u_{d}\right\|_{\infty}<d_{\max }$ with $\|\cdot\|_{\infty}$ the vector infinity-norm and $d_{\max }$ is an unknown positive constant.

The control objective is summarized as follows: synthesize a robust attitude controller in the ASMC framework such that the system states in (10) can be stabilized without a prior knowledge of $d_{\max }$. Moreover, the switching gain generated by the ASMC algorithm should be as small as possible without affecting the system stability.

2.2. Current ASMC Design. In this subsection, current ASMC design will be briefly reviewed and applied to the control problem under consideration. According to the ASMC design principle in [19], a proper sliding function $S \in \mathbb{R}^{3}$ is firstly defined as

$$
S=\omega_{e}+\Lambda \frac{M^{T}}{m} \sigma_{e}
$$

where $\Lambda=\operatorname{diag}\left(\lambda_{1}, \lambda_{2}, \lambda_{3}\right) \in \mathbb{R}^{3 \times 3}$ is the sliding function gain matrix with its elements $\lambda_{i}>0(i=1,2,3)$. As usual in the SMC technique, the SMC-based attitude control law takes the form of $u=u_{\mathrm{eq}}+u_{\mathrm{sw}}$, where $u_{\mathrm{eq}}$ is the equivalent control component derived from $\dot{S}=0$ (or from $\widehat{J} \dot{S}=0$ actually) in the absence of the lumped uncertainty and $u_{\mathrm{sw}}$ is the discontinuous switching control part dealing with the lumped uncertainty. Then, it is easy to obtain the following SMC-based attitude controller by choosing the Lyapunov function as $V=S^{T} \widehat{J} S / 2$ :

$$
\begin{aligned}
u= & \left(\omega_{e}+R \omega_{d}\right)^{\times} \widehat{J}\left(\omega_{e}+R \omega_{d}\right)-\widehat{J} \omega_{e}^{\times} R \omega_{d} \\
& +\widehat{J} R \dot{\omega}_{d}-\widehat{J} \Lambda \frac{\mathrm{d}}{\mathrm{d} t}\left(\frac{M^{T}}{m} \sigma_{e}\right)-\Gamma \operatorname{sgn}(S),
\end{aligned}
$$

where $(\mathrm{d} / \mathrm{d} t)(\cdot)$ denotes the time derivative and $\Gamma=\operatorname{diag}\left(\gamma_{1}\right.$, $\left.\gamma_{2}, \gamma_{3}\right)$ is the switching gain matrix with its elements $\gamma_{i}(i=$ $1,2,3)$ at least larger than $d_{\max }$ to guarantee the stability.

When $d_{\text {max }}$ is unavailable in advance, the ASMC technique can be employed by producing a negative derivative of the following enhanced Lyapunov function [19]:

$$
V=\frac{1}{2} S^{T} \widehat{J} S+\frac{1}{2 c} \widetilde{d}^{2}
$$

where $\tilde{d}=\widehat{d}-d_{\max }$ is the estimation error with $\widehat{d}$ the estimation of $d_{\max }$ and $c>0$ is the adaptive gain. Then, the ASMC-based attitude control law can be obtained by simply replacing the switching gain matrix $\Gamma$ with $\widehat{d}$ as

$$
\begin{gathered}
u=\left(\omega_{e}+R \omega_{d}\right)^{\times} \widehat{J}\left(\omega_{e}+R \omega_{d}\right)-\widehat{J} \omega_{e}^{\times} R \omega_{d} \\
+\widehat{J} R \dot{\omega}_{d}-\widehat{J} \Lambda \frac{\mathrm{d}}{\mathrm{d} t}\left(\frac{M^{T}}{m} \sigma_{e}\right)-\widehat{d} \operatorname{sgn}(S), \\
\widehat{d}=c \int_{0}^{t}\|S\|_{1} \mathrm{~d} \tau .
\end{gathered}
$$

Remark 1. By inspecting the relationship between the Jacobian matrix and the MRP error and using the fact that $\sigma_{e}^{\times} \sigma_{e}=$ 0 , one can get

$$
\frac{M^{T} \sigma_{e}}{m}=\frac{4 \sigma_{e}}{1+\left\|\sigma_{e}\right\|^{2}},
$$

then, one has

$$
\frac{\mathrm{d}}{\mathrm{d} t}\left(\frac{M^{T} \sigma_{e}}{m}\right)=\frac{4 M^{T}-2 \sigma_{e} \sigma_{e}^{T}}{1+\left\|\sigma_{e}\right\|^{2}} \omega_{e},
$$

which implies that the ASMC algorithm in (14) can be constructed without a complex differential calculus.

\section{Main Results}

3.1. Overadaptation Problem in Current ASMC Design. From (15), one can conclude that the philosophy of the ASMC technique lies in adjusting the switching gain according to the departure from the sliding surface, which is characterized by the 1-norm of the sliding function. In order to give a better estimation of the upper bound on the lumped uncertainty, it is desired that the sliding surface departure is totally or at least 
mainly induced by the lumped uncertainty. However, due to the fact that the integral action starts at the very beginning of the control action, it is obvious that the departure caused by any factors will result in an increase of the switching gain. Furthermore, using the two-stage phase trajectory concept in $\mathrm{SMC}$, one can divide the switching gain adaptation into two parts, that is,

$$
\widehat{d}=c \int_{0}^{t_{r}}\|S\|_{1} \mathrm{~d} \tau+c \int_{t_{r}}^{t}\|S\|_{1} \mathrm{~d} \tau
$$

with $t_{r}$ the arrival time at the sliding surface. Equation (18) indicates that the adaptation process also includes two parts, the reaching phase adaptation and the sliding phase adaptation. It is very important to note that the sliding surface departure in the reaching phase is mainly caused by the initial system error but not the lumped uncertainty. Hence, with the existence of such an unrelated adaptation, the switching gain is undoubtedly overestimated, which is the underlying reason for the overadaptation problem in current ASMC design and has not been fully addressed in the literature.

\subsection{ASMC Design without Switching Gain Overadaptation.} The overadaptation problem may consequently lead to deteriorations in system performance. As is well known, the chattering level of discontinuous SMC laws is proportional to the switching gain. Therefore, systems employing current ASMC design may present a serious chattering phenomenon in control signal. On the other hand, when continuous approximation technique, for example, the boundary layer method, is used to soften the chattering phenomenon, there will be an undesirable large-amplitude jump in control signal when the system states are changing inside the boundary layer. Hence, in this subsection, such a problem will be addressed by exploiting the global sliding mode feature of integral sliding mode control (ISMC) technique for the ASMC algorithm derivation. Before moving on, an ISMC-based attitude controller is presented to demonstrate the motivation of the ASMC design without switching gain overadaptation.

First, a nominal feedback control law is put forward for the following nominal attitude tracking system in the absence of the lumped uncertainty:

$$
\begin{gathered}
\widehat{J} \dot{\omega}_{e}=-\left(\omega_{e}+R \omega_{d}\right)^{\times} \widehat{J}\left(\omega_{e}+R \omega_{d}\right)+\widehat{J} \omega_{e}^{\times} R \omega_{d}-\widehat{J} R \dot{\omega}_{d}+u_{n}, \\
\dot{\sigma}_{e}=M \omega_{e},
\end{gathered}
$$

where $u_{n} \in \mathbb{R}^{3 \times 3}$ stands for the nominal control, which is selected as

$$
\begin{aligned}
u_{n}= & \left(\omega_{e}+R \omega_{d}\right)^{\times} \widehat{J}\left(\omega_{e}+R \omega_{d}\right) \\
& -\widehat{J} \omega_{e}^{\times} R \omega_{d}+\widehat{J} R \dot{\omega}_{d}-k_{d} \widehat{J} \omega_{e}-k_{p} \widehat{J} \sigma_{e},
\end{aligned}
$$

with $k_{d}>0$ and $k_{p}>0$.

Consider the Lyapunov candidate function as

$$
V=\frac{1}{2} \omega_{e}^{T} \omega_{e}+2 k_{p} \ln ^{1+\sigma_{e}^{T} \sigma_{e}} .
$$

By taking the time derivative of the Lyapunov function and substituting the nominal control law, one has

$$
\begin{aligned}
\dot{V} & =\omega_{e}^{T} \widehat{J}^{-1} \widehat{J} \dot{\omega}_{e}+4 k_{p} \frac{\sigma_{e}^{T} M \omega_{e}}{1+\sigma_{e}^{T} \sigma_{e}} \\
& =\omega_{e}^{T} \widehat{J}^{-1}\left(-k_{d} \widehat{J} \omega_{e}-k_{p} \widehat{J} \sigma_{e}\right)+k_{p} \sigma_{e}^{T} \omega_{e} \\
& =-k_{d} \omega_{e}^{T} \omega_{e},
\end{aligned}
$$

where the fact $k_{p} \omega_{e}^{T} \sigma_{e}=k_{p} \sigma_{e}^{T} \omega_{e}$ has been used. According to Barbalat lemma, one has $\lim _{t \rightarrow \infty} \omega_{e}=0$. By substituting the nominal control law into the error attitude dynamics, one can conclude $\lim _{t \rightarrow \infty} \sigma_{e}=0$. Therefore, the nominal system is asymptotically stable. Moreover, the system response is governed by the following equation:

$$
\dot{\omega}_{e}+k_{d} \omega_{e}+k_{p} \sigma_{e}=0
$$

By taking the second order derivative of $\omega_{e}$, the following classical second order system can be obtained:

$$
\ddot{\omega}_{e}+k_{d} \dot{\omega}_{e}+k_{p} M \omega_{e}=0
$$

which is convenient for selecting $k_{d}$ and $k_{p}$ to achieve the desired performance.

Then, a proper sliding function should be established to make the equivalent control component of the ISMC law same as the predefined nominal controller in (20). To this end, define the integral sliding function $S_{I} \in \mathbb{R}^{3}$ as

$$
S_{I}=g(x)+Z \text {. }
$$

Here $g(x) \in \mathbb{R}^{3}$ is selected as the conventional sliding function $S$ defined in (11), which can be replaced by any proper functions. $Z \in \mathbb{R}^{3}$ is the integral term to be determined. Considering the fact that the equivalent control component is derived from $\dot{S}_{I}=0$, one can select the Lyapunov candidate function as

$$
V=\frac{1}{2} S_{I}^{T} \widehat{J}_{I}
$$

Taking the time derivative of the Lyapunov function yields

$$
\begin{aligned}
\dot{V}= & S_{I}^{T} \widehat{J} \dot{S}_{I} \\
= & S_{I}^{T}\left[\widehat{J} \dot{\omega}_{e}+\widehat{J} \Lambda \frac{\mathrm{d}}{\mathrm{d} t}\left(\frac{M^{T} \sigma_{e}}{m}\right)+\widehat{J} \dot{Z}\right] \\
= & S_{I}^{T}\left[u_{n}-\left(\omega_{e}+R \omega_{d}\right)^{\times} \widehat{J}\left(\omega_{e}+R \omega_{d}\right)+\widehat{J} \omega_{e}^{\times} R \omega_{d}\right. \\
& \left.\quad-\widehat{J} R \dot{\omega}_{d}+\widehat{J} \Lambda \frac{\mathrm{d}}{\mathrm{d} t}\left(\frac{M^{T} \sigma_{e}}{m}\right)+\widehat{J} \dot{Z}\right]+S_{I}^{T}\left(u_{\mathrm{sw}}+u_{d}\right) \\
= & S_{I}^{T}\left[\widehat{J} \Lambda \frac{\mathrm{d}}{\mathrm{d} t}\left(\frac{M^{T} \sigma_{e}}{m}\right)+\widehat{J} \dot{Z}-k_{d} \widehat{J} \omega_{e}-k_{p} \widehat{J} \sigma_{e}\right] \\
& +S_{I}^{T}\left(u_{\mathrm{sw}}+u_{d}\right) .
\end{aligned}
$$


It is natural to choose $\dot{Z}=k_{d} \omega_{e}+k_{p} \sigma_{e}-\Lambda \frac{\mathrm{d}}{\mathrm{d} t}\left(M^{T} \sigma_{e} / m\right)$ and $u_{\mathrm{sw}}=-\Gamma \operatorname{sgn}\left(S_{I}\right)$, where the switching gain matrix $\Gamma$ is the same as that in (12). Hence, the ISMC-based attitude controller is presented as

$$
\begin{aligned}
u= & u_{n}+u_{\mathrm{sw}} \\
= & \left(\omega_{e}+R \omega_{d}\right)^{\times} \widehat{J}\left(\omega_{e}+R \omega_{d}\right)-\widehat{J} \omega_{e}^{\times} R \omega_{d} \\
& +\widehat{J} R \dot{\omega}_{d}-k_{d} \widehat{J} \omega_{e}-k_{p} \widehat{J} \sigma_{e}-\Gamma \operatorname{sgn}\left(S_{I}\right) .
\end{aligned}
$$

Following the same line of conventional SMC, one can conclude that the sliding mode will start in finite time, that is, $S_{I}=0$ for $t \in\left[t_{r}, \infty\right)$. When $S_{I}=0, \dot{S}_{I}=0$ also holds, which indicates $\dot{\omega}_{e}=-k_{d} \omega_{e}-k_{p} \sigma_{e}$. Then, the stability analysis can be completed by choosing the Lyapunov function same as (21).

Moreover, a useful lemma can be obtained as stated in the following.

Lemma 2. For the attitude tracking system described by (10), by defining the integral sliding function as (25) and adopting the ISMC law in (28), a global sliding mode will be achieved if the initial value of $Z$ is selected as $Z(0)=-S(0)$.

Proof. The condition $Z(0)=-S(0)$ guarantees that the initial value of the integral sliding function is zero, that is, $S_{I}(0)=0$. Hence, the initial value of the Lyapunov function in (26) is also zero. From the foregoing derivations, a negative definite derivative of the Lyapunov function can be produced by the ISMC algorithm in (28). Then, one has $V \leq V(0)=0$. According to the definition of the positive definite Lyapunov function, that is, $V \geq 0$, one can easily conclude that $V \equiv 0$, which indicates that $S_{I} \equiv 0$ for for all $t \in[0, \infty)$.

Lemma 2 shows the excellent feature of the ISMC technique, which indicates that the ISMC cannot only guarantee the asymptotic stability of the entire system but also can keep the system states moving on the sliding surface throughout the control action. Such a feature directly implies an improvement of the robustness against matched model uncertainty and external disturbance. Moreover, the system performance can also be enhanced, as the nominal controller is responsible for the system response. The related phase trajectory of ISMC is illustrated in Figure 1, and the corresponding 1-norm of the sliding function response is shown in Figure 2, as compared with conventional SMC.

From Figure 1, one can clearly see that the reaching phase in conventional SMC is eliminated in ISMC and the system states are constrained on the sliding surface all the time. Furthermore, as shown in Figure 2, it is obvious that the 1-norm of the sliding function characterizing the sliding surface departure is significantly reduced as compared with conventional SMC. Under the assumption of ideal sliding mode, there will be no departure from the sliding surface in ISMC. Therefore, if the ASMC algorithm is developed based on the ISMC technique, the unrelated sliding surface departure induced by the initial tracking error would be removed, and consequently a more accurate estimation of the switching gain can be generated, which is the motivation of the ASMC design without switching gain overadaptation.
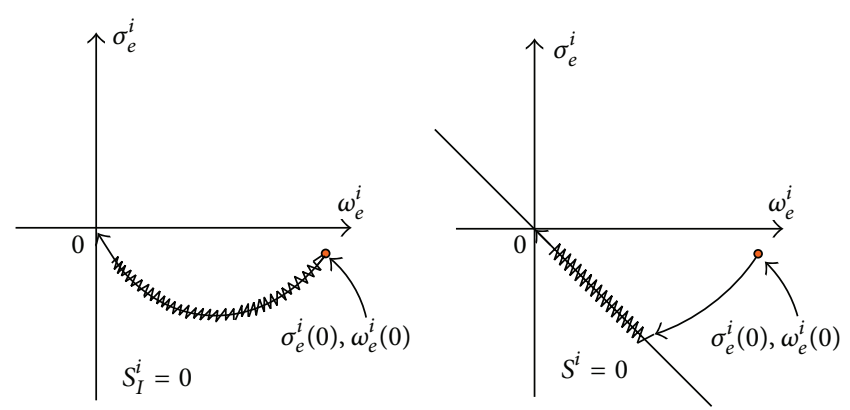

FIGURE 1: Comparison of the phase trajectories of ISMC and SMC.

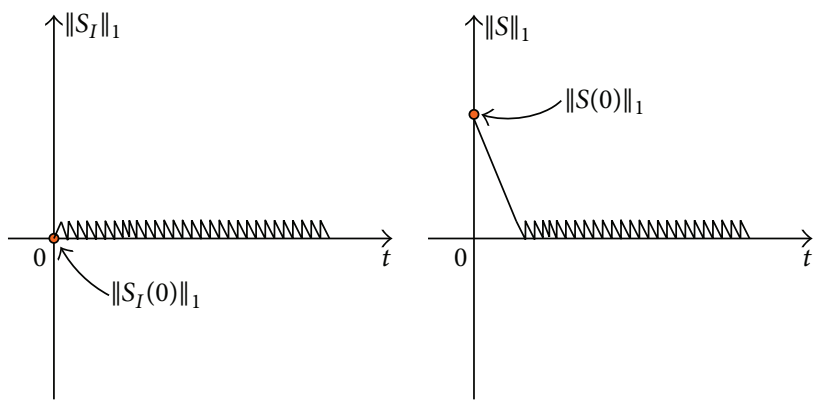

FIGURE 2: 1-norm of sliding function comparison between ISMC and SMC.

The ISMC-based ASMC algorithm is now presented as

$$
\begin{gathered}
u=\left(\omega_{e}+R \omega_{d}\right)^{\times} \widehat{J}\left(\omega_{e}+R \omega_{d}\right)-\widehat{J} \omega_{e}^{\times} R \omega_{d} \\
+\widehat{J} R \dot{\omega}_{d}-k_{d} \widehat{J} \omega_{e}-k_{p} \widehat{J} \sigma_{e}-\widehat{d} \operatorname{sgn}\left(S_{I}\right), \\
\widehat{d}=c \int_{0}^{t}\left\|S_{I}\right\|_{1} \mathrm{~d} \tau .
\end{gathered}
$$

Then, one can obtain the following theorem.

Theorem 3. For the attitude tracking system described by (10), by defining the integral sliding function as (25) and utilizing the ISMC-based ASMC algorithm in (29) and (30), the integral sliding function is asymptotically stable, that is, $\lim _{t \rightarrow \infty} S_{I}=0$.

Proof. Consider the Lyapunov function as

$$
V=\frac{1}{2} S_{I}^{T} \widehat{J} S_{I}+\frac{1}{2 c} \widetilde{d}^{2}
$$

By taking the time derivative of the above Lyapunov function along closed-loop system trajectory, one has

$$
\begin{aligned}
\dot{V} & =S_{I}^{T} \widehat{\mathcal{J}} \dot{S}_{I}+\frac{\tilde{d} \dot{\vec{d}}}{c} \\
& =S_{I}^{T}\left[u_{d}-\widehat{d} \operatorname{sgn}\left(S_{I}\right)\right]+\left(\widehat{d}-d_{\max }\right)\left\|S_{I}\right\|_{1} \\
& =S_{I}^{T} u_{d}-d_{\max }\left\|S_{I}\right\|_{1} \\
& \leq-\left(d_{\max }-\left\|u_{d}\right\|_{\infty}\right)\left\|S_{I}\right\|_{1} .
\end{aligned}
$$


Let $\chi=\left(d_{\max }-\left\|u_{d}\right\|_{\infty}\right)\left\|S_{I}\right\|_{1}$, which is uniformly continuous. By integrating the above equation from 0 to $t$, one has

$$
\begin{aligned}
\int_{0}^{t} \dot{V} \mathrm{~d} \tau \leq-\int_{0}^{t} \chi \mathrm{d} \tau & \Longrightarrow V(0) \geq V(t)+\int_{0}^{t} \chi \mathrm{d} \tau \\
& \Longrightarrow \int_{0}^{t} \chi \mathrm{d} \tau \leq V(0)
\end{aligned}
$$

Taking the limits as $t \rightarrow \infty$ gives

$$
\lim _{t \rightarrow \infty} \int_{0}^{t} \chi \mathrm{d} \tau \leq V(0) \leq \infty
$$

According to Barbalat lemma, one has

$$
\lim _{t \rightarrow \infty} \chi \longrightarrow 0
$$

Considering the fact that $\left\|u_{d}\right\|_{\infty}<d_{\max }$, the conclusion can be easily obtained.

Furthermore, the asymptotic stability of the entire system can be guaranteed by an alternative intuitive explanation provided for current ASMC design in [26]. According to the adaptive laws in (15) and (30), one can see that the switching gain will keep increasing until the sliding mode occurs. Once the switching gain increases up to a value large enough to suppress the lumped uncertainty, the reaching condition is satisfied and the sliding mode will start in finite time. Consequently, the asymptotic stability of the closedloop system can be ensured.

Remark 4. According to the preceding design, the influence induced by the initial error is totally removed in the switching gain adaptation. Therefore, the resulting switching gain is much smaller than C-ASMC. As is well known, the arrival time at the sliding surface is inversely proportional to the switching gain in conventional SMC. Therefore, if a small switching gain is chosen, the system response will be slowed down drastically. Whereas, the system performance controlled by I-ASMC is totally governed by the dynamics of nominal system, where the nominal control law determines the response speed rather than the switching gain. Therefore, when a small switching gain is used in the discontinuous control component, the system performance will not be obviously affected if a proper nominal control is selected in the I-ASMC design.

\section{Simulation Results}

In this section, a numerical simulation is employed to test the validity of the proposed strategy. Current ASMC algorithm in (14) and (15) and the ISMC-based ASMC algorithm in (29) and (30) will be applied to the control problem under consideration and are, respectively, referred to as C-ASMC and I-ASMC for clarity.

The nominal inertia matrix used for the controllers is $\widehat{J}=$ $\operatorname{diag}(950,600,360)$, and the uncertainty on the inertia matrix is about $10 \%$ of the nominal value. The external disturbance is

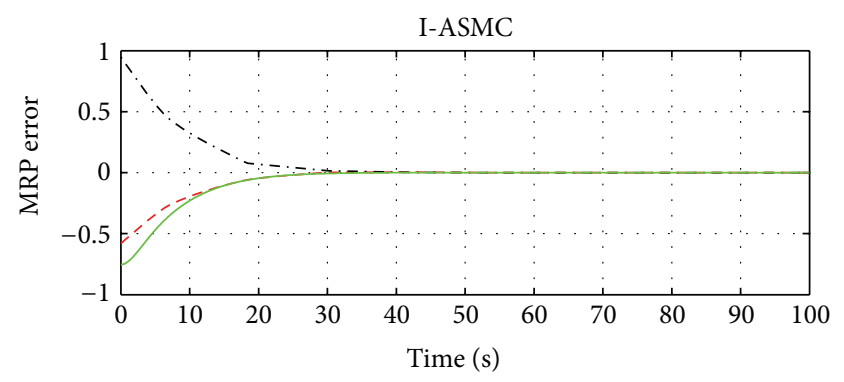

(a)

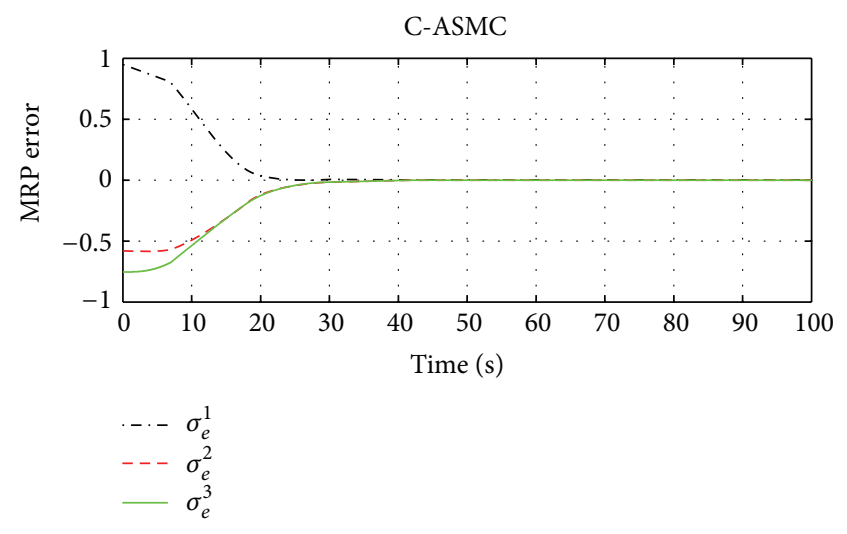

(b)

FIGURE 3: Comparison of MRP error responses.

assumed as $d=[\sin 0.1 t, 2 \cos 0.1 t, 3 \sin 0.2 t]^{T} \times 10^{-3}$ (N.m). The desired attitude trajectory is described according to the attitude kinematics in (2), where the angular velocity is $\omega_{d}=[\sin 0.05 t, 5 \sin 0.02 t, 3 \sin 0.02 t]^{T} \times 10^{-3}(\mathrm{rad} / \mathrm{s})$ and the initial MRP is $\sigma_{d}(0)=[-0.2,0.3,0.1]^{T}$. The objective for the spacecraft is to track such a given attitude trajectory from its initial states, $\sigma(0)=[0.3,-0.4,-0.5]^{T}$ and $\omega(0)=$ $[0,0,0]^{T}(\mathrm{rad} / \mathrm{s})$. For comparison, same control parameters are used, where the sliding function gain matrix is $\Lambda=$ $0.2 I_{3}$, the adaptive gain is $c=2$, and the initial value of $\widehat{d}$ is zero. Other parameters are adjusted such that those ASMC algorithms possess a similar setting time; that is, the spacecraft is required to track the given attitude trajectory from $t \approx 30$ (sec), where $k_{d}=0.3$ and $k_{p}=0.1$ for I-ASMC.

To begin with, simulation test is conducted using discontinuous ASMC laws, where the simulation sample time is set to 0.001 ( $\mathrm{sec}$ ) to approximately achieve the ideal sliding mode. The simulation results are shown in Figures 3-8, where the superscript $(\cdot)^{i}(i=1,2,3)$ denotes the triaxial component of related vectors.

Figures 3 and 4 depict the attitude tracking evolutions controlled by those two ASMC algorithms, where the comparison of the MRP error responses is shown in Figure 3 and the angular velocity error responses are compared in Figure 4. It can be seen from Figures 3 and 4 that both ASMC algorithms have successfully accomplished the attitude tracking without a prior knowledge of $d_{\max }$. Moreover, from Figure 3, one can see that the MRP error convergence rate controlled by I-ASMC was faster than C-ASMC. Such a performance 


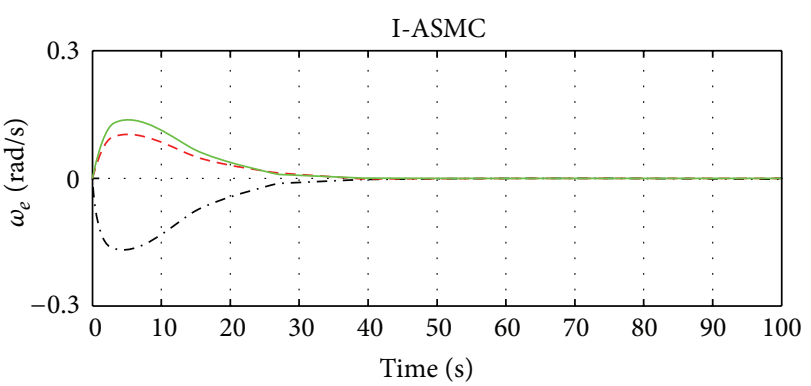

(a)

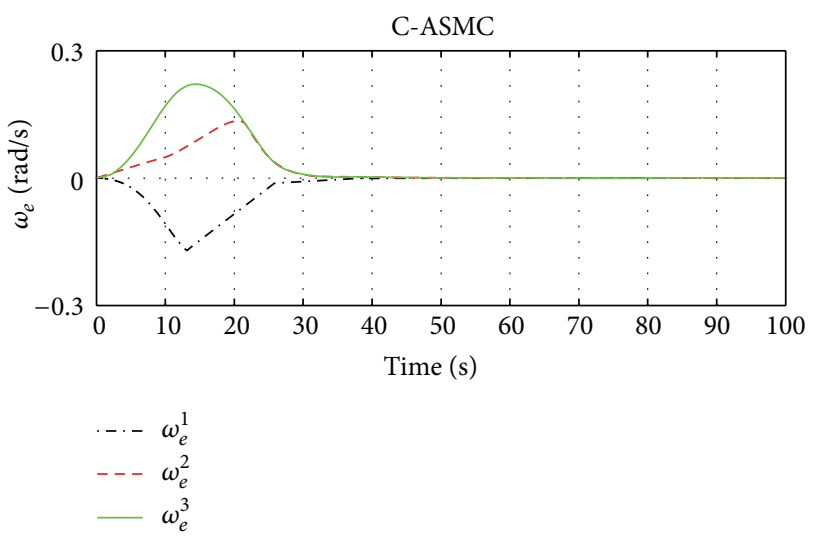

(b)

FIGURE 4: Comparison of angular velocity error responses.
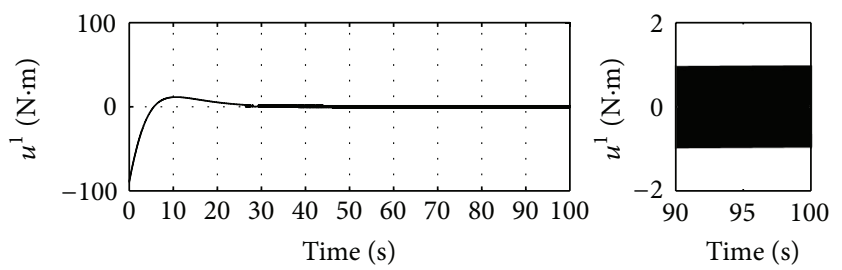

(a)
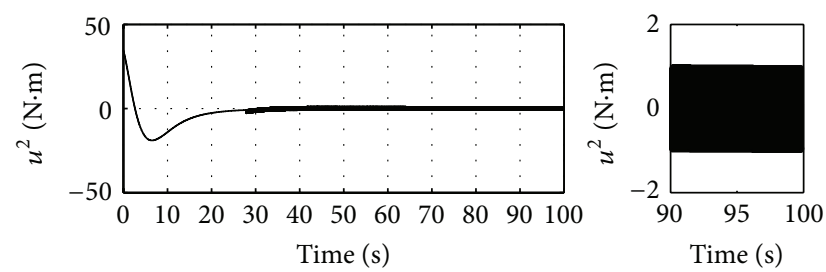

(b)
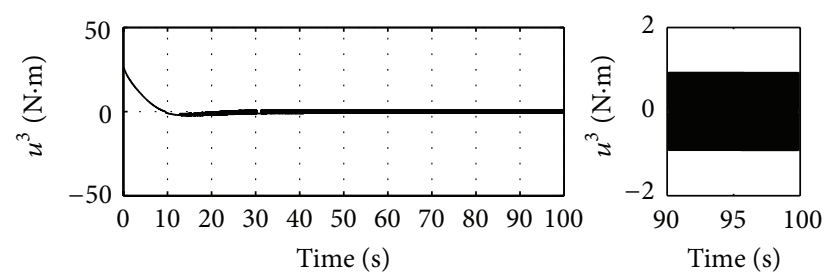

(c)

FIGURE 5: Control torque commands computed from I-ASMC.
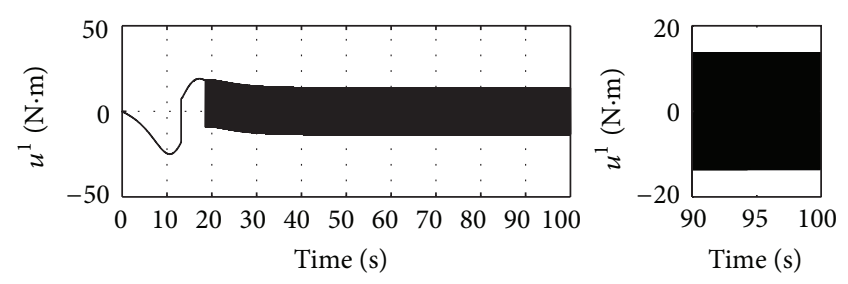

(a)
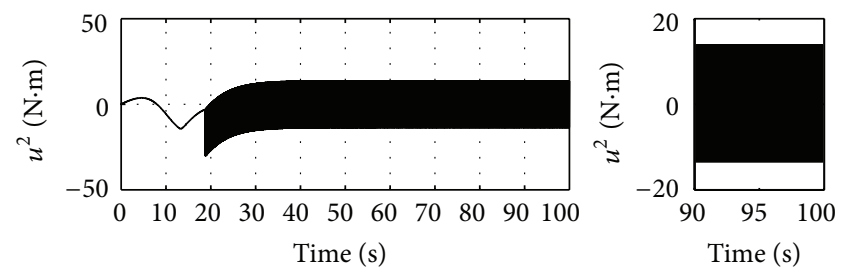

(b)
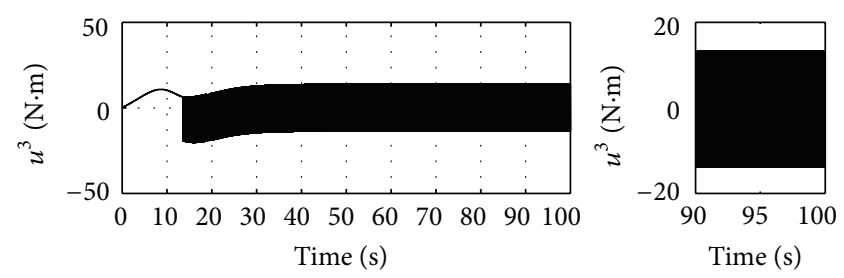

(c)

FIGURE 6: Control torque commands computed from C-ASMC.

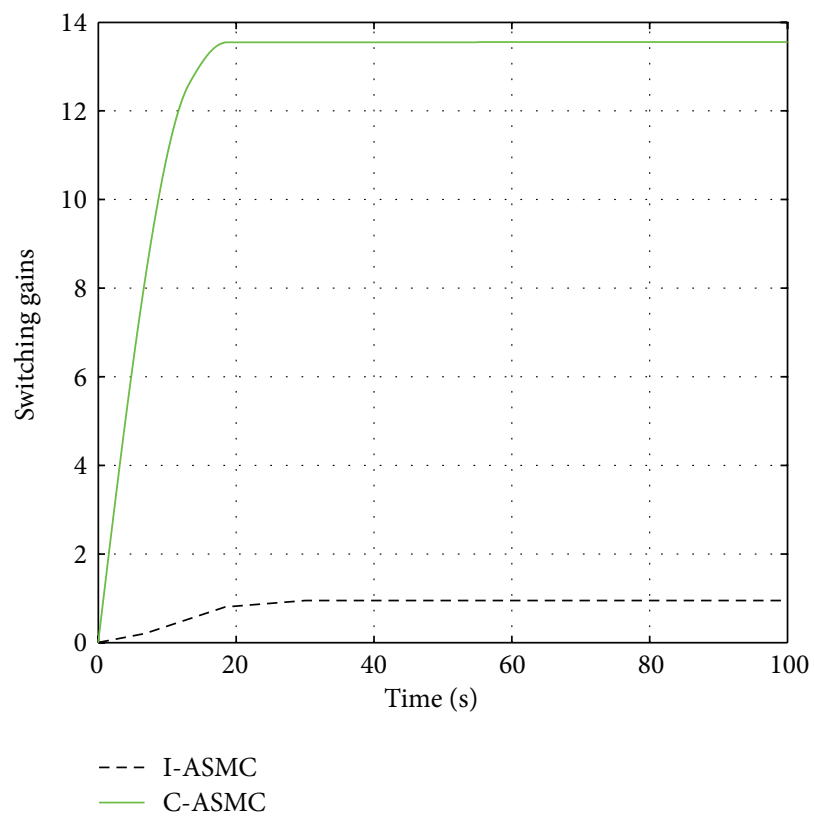

FIGURE 7: Comparison of switching gains generated by the ASMC algorithms.

improvement is owing to the flexibility of ISMC technique, where the system performance is dominated by the nominal control component. Whereas, in conventional SMC, the system performance in the reaching phase cannot be specified.

Figures 5 and 6 show the control torque commands calculated according to those ASMC algorithms, as well as 


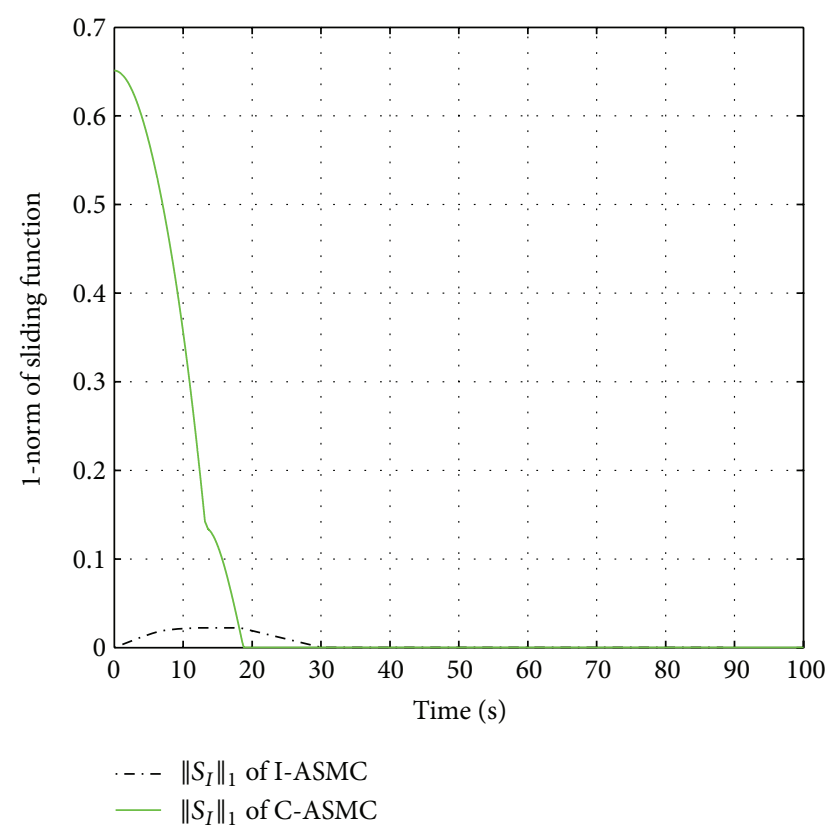

FIGURE 8: Comparison of the 1-norm of sliding function responses.

the enlarged diagrams in the steady-state regime. Compared with that computed by C-ASMC in Figure 6, one can clearly see that the chattering phenomenon was significantly reduced by I-ASMC and a lower-chattering control signal is achieved, which verifies the effectiveness of the chattering suppression ability of the proposed method. Such an advantage can also be validated in Figure 7, where the switching gain generated by I-ASMC was about 0.95 while $\widehat{d} \approx 13.5$ for C-ASMC. Due to the fact that the chattering level is directly related to the switching gain, the significant difference in the control chattering is explained.

Moreover, the sliding function responses in terms of 1norm are compared in Figure 8. From Figure 8, one can see that the sliding function in I-ASMC started from zero then was driven away from zero by the lumped uncertainty, as the switching gain was not large enough to suppress the lumped uncertainty. Along with the increase of the switching gain, the sliding function would converge to zero again. Whereas, the sliding function in C-ASMC possessed a nonzero initial value, which may be very large when a large angle attitude tracking is considered. Based on the philosophy of ASMC design, the switching gain produced by C-ASMC was undoubtedly much bigger than that of I-ASMC, which has been proven in Figure 7.

Then, for implementation in practice, simulation test is conducted when the boundary layer technique is used to soften the chattering phenomenon. Under this circumstance, the simulation sample time is selected as $0.2(\mathrm{sec})$ and the boundary layer thickness is chosen as 0.002 . In order to get bounded switching gains, the adaptive laws in (15) and (30) are modified as

$$
\widehat{d}=c \int_{0}^{t}\left(\|S\|_{1}-\kappa \widehat{d}\right) \mathrm{d} \tau
$$

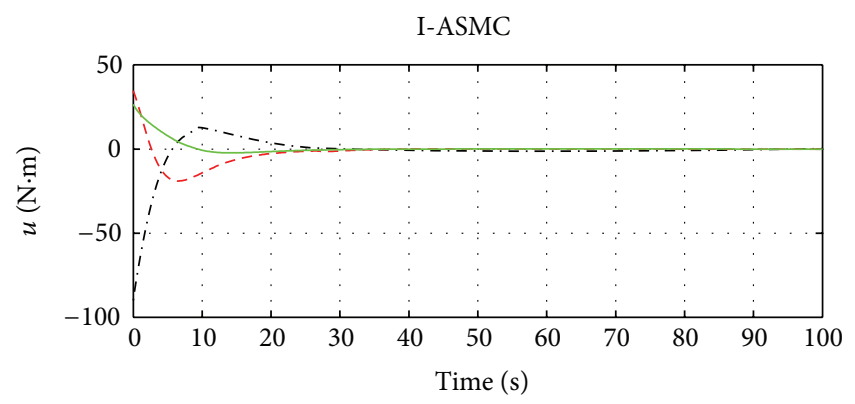

(a)

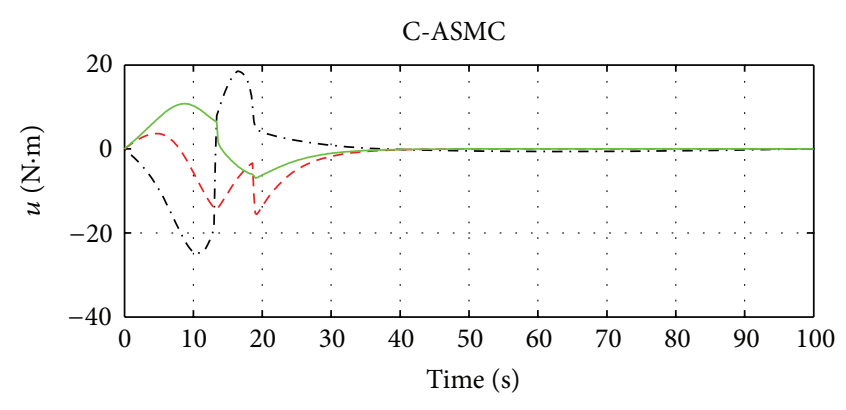

$$
\begin{aligned}
& --u^{1} \\
& --u^{2} \\
& -u^{3}
\end{aligned}
$$

(b)

FIGURE 9: Comparison of control torque commands computed from the ASMC algorithms.

where $\kappa=0.00001$ and $S$ can be replaced by $S_{I}$ in (25) for IASMC. As the attitude tracking evolutions are quite similar to the discontinuous case, the comparisons of MRP error, angular velocity error, and sliding function are omitted here. Only the control torque commands generated by those ASMC algorithms after continuous approximation are illustrated in Figure 9.

As seen from Figure 9, large-amplitude jumps existed in the control signal computed by C-ASMC. Take the 1st axis for example, the control torque command instantaneously increased from -20 (N.m) to $10(\mathrm{~N} . \mathrm{m})$ at $t \approx 13$ (sec), which is undesirable for the actuator servo control system. Such a problem is also caused by the overestimated switching gain when the sliding function is changing within the boundary layer. While for I-ASMC, the control torque command was fairly smooth and is more suitable for engineering applications.

\section{Conclusion}

In this paper, the overadaptation problem in current ASMC design has been addressed in the context of attitude control for a rigid spacecraft involving inertia matrix uncertainty and external disturbance. The underlying reason for the overadaptation problem is analyzed by inspecting the switching gain adaptation mechanism. An improved ASMC design principle without switching gain overadaptation is then presented by using the global sliding mode feature of the ISMC 
technique. The presented method can be directly applied to many other nonlinear systems without any modifications. The validity of the proposed strategy has been proven by both theoretical analysis and simulation results.

\section{Acknowledgments}

The authors acknowledge the financial support from Major State Basic Research Development Program under Grant 2012CB720000, National Natural Science Foundation of China under Grant 61104153, and Technology Innovation Program of Beijing Institute of Technology under Grant CX0428.

\section{References}

[1] S. R. Vadali, "Variable-structure control of spacecraft largeangle maneuvers," Journal of Guidance, Control, and Dynamics, vol. 9, no. 2, pp. 235-239, 1986.

[2] Y. P. Chen and S. C. Lo, "Sliding-mode controller design for spacecraft attitudetracking maneuvers," IEEE Transactions on Aerospace and Electronic Systems, vol. 29, no. 4, pp. 1328-1333, 1993.

[3] S. C. Lo and Y. P. Chen, "Smooth sliding-mode control for spacecraft attitude tracking maneuvers," Journal of Guidance, Control, and Dynamics, vol. 18, no. 6, pp. 1345-1349, 1995.

[4] J. D. Bošković, S. M. Li, and R. K. Mehra, "Robust adaptive variable structurecontorl of spacecraft uner control input saturation"' Journal of Guidance, Control, and Dynamics, vol. 24, no. 1, pp. 14-22, 2001.

[5] F. K. Yeh, H. H. Chien, and L. C. Fu, "Design of optimal midcourse guidance sliding-mode control for missiles with TVC," IEEE Transactions on Aerospace and Electronic Systems, vol. 39, no. 3, pp. 824-837, 2003.

[6] J. D. Bošković, S. M. Li, and R. K. Mehra, "Robust tracking control design for spacecraftunder control input saturation," Journal of Guidance, Control, and Dynamics, vol. 27, no. 4, pp. 627-633, 2004.

[7] H. Bang, C. K. Ha, and J. H. Kim, "Flexible spacecraft attitude maneuver by application of sliding mode control," Acta Astronautica, vol. 57, no. 11, pp. 841-850, 2005.

[8] F. K. Yeh, "Sliding-mode adaptive attitude controller design for spacecrafts with thrusters," IET Control Theory and Applications, vol. 4, no. 7, pp. 1254-1264, 2010.

[9] Z. Zhu, Y. Q. Xia, and M. Y. Fu, "Adaptive sliding mode control for attitude stabilizationwith actuator saturation,"' IEEE Transactions on Industrial Electronics, vol. 58, no. 10, pp. 4898-4907, 2011.

[10] A. W. D. Thomas and S. R. Hebertt, "Variable-structure control of spacecraft attitude maneuvers," Journal of Guidance, Control, and Dynamics, vol. 11, no. 3, pp. 262-270, 1988.

[11] J. L. Crassidis and F. L. Markley, "Sliding mode control using modified Rodrigues parameters," Journal of Guidance, Control, and Dynamics, vol. 19, no. 6, pp. 1381-1383, 1996.

[12] C. Binglong, L. Xiangdong, and C. Zhen, "Exponential timevarying sliding mode control for large angle attitude eigenaxis maneuver of rigid spacecraft," Chinese Journal of Aeronautics, vol. 23, no. 4, pp. 447-453, 2010.

[13] S. A. Kowalchuk and C. D. Hall, "Spacecraft attitude sliding mode controller using reaction wheels," in Proceedings of
AIAA/AAS Astrodynamics Specialist Conference and Exhibit, pp. 1-28, Honolulu, Hawaii, USA, August 2008.

[14] B. L. Cong, X. D. Liu, and Z. Chen, "Distributed attitude synchronization of formation flying via consensus-based virtual structure," Acta Astronautica, vol. 68, no. 11-12, pp. 1973-1986, 2011.

[15] H. Elmali and N. Olgac, "Satellite attitude control via sliding mode with perturbationestimation,"' IEE Proceedings Control Theory \& Applications, vol. 143, no. 3, pp. 276-282, 1996.

[16] J. Kim, J. Kim, and J. L. Crassidis, "Disturbance accommodating sliding modecontroller for spacecraft attitude maneuvers," in Proceedings of the 13th International Symposiumon Space Flight Dynamics, pp. 119-131, Greenbelt, Md, USA, 1998.

[17] U. Jørgensen and J. T. Gravdahl, "Observer based sliding mode attitude control:theoretical and experimental results," Modeling, Identification and Control, vol. 31, no. 1, pp. 1-9, 2010.

[18] D. S. Yoo and M. J. Chung, "A variable structure control with simple adaptation laws for upper bounds on the norm of the uncertainties," IEEE Transactions on Automatic Control, vol. 37, no. 6, pp. 860-865, 1992.

[19] F. J. Lin, S. L. Chiu, and K. K. Shyu, "Novel slidign mode controller for synchronousmotor drive," IEEE Transactions on Aerospace and Electronic Systems, vol. 34, no. 2, pp. 532-542, 1998.

[20] R. J. Wai, "Adaptive sliding-mode control for induction servomotor drive," IEE Proceedings: Electric Power Applications, vol. 147 , no. 6, pp. 553-562, 2000.

[21] J. S. Souder and J. K. Hedrick, "Adaptive sliding mode control of air-fuel ratio in internal combustion engines," International Journal of Robust and Nonlinear Control, vol. 14, no. 6, pp. 525541, 2004.

[22] Y. J. Huang, T. C. Kuo, and S. H. Chang, "Adaptive slidingmode control for nonlinearsystems with uncertain parameters," IEEE Transactions on Systems, Man, and Cybernetics, Part B: Cybernetics, vol. 38, no. 2, pp. 534-539, 2008.

[23] M. Shahravi, M. Kabganian, and A. Alasty, "Adaptive robust attitude control of a flexible spacecraft," International Journal of Robust and Nonlinear Control, vol. 16, no. 6, pp. 287-302, 2006.

[24] Q. L. Hu, "Robust adaptive sliding mode attitude control and vibration dampingof flexible spacecraft subject to unknown disturbance and uncertainty," Transactions of the Institute of Measurement and Control, vol. 33, no. 7, pp. 1-12, 2011.

[25] P. Ioannou and B. Fidan, Adaptive Control Tutorial, vol. 11 of Advances in Design and Control, SIAM, Philadelphia, Pa, USA, 1st edition, 2006.

[26] F. Plestan, Y. Shtessel, V. Brégeault, and A. Poznyak, "New methodologies for adaptive sliding mode control," International Journal of Control, vol. 83, no. 9, pp. 1907-1919, 2010. 


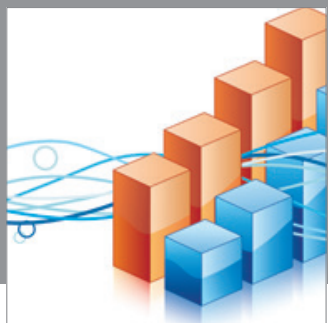

Advances in

Operations Research

mansans

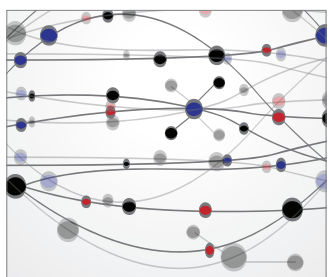

The Scientific World Journal
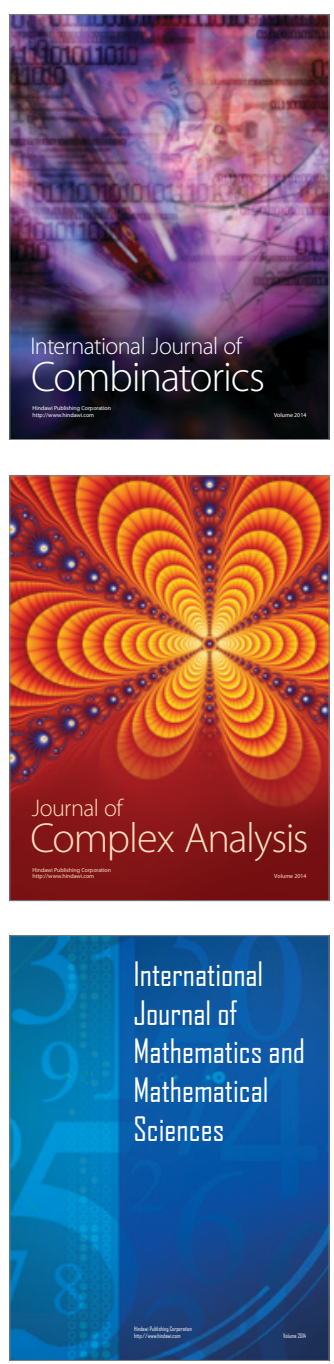
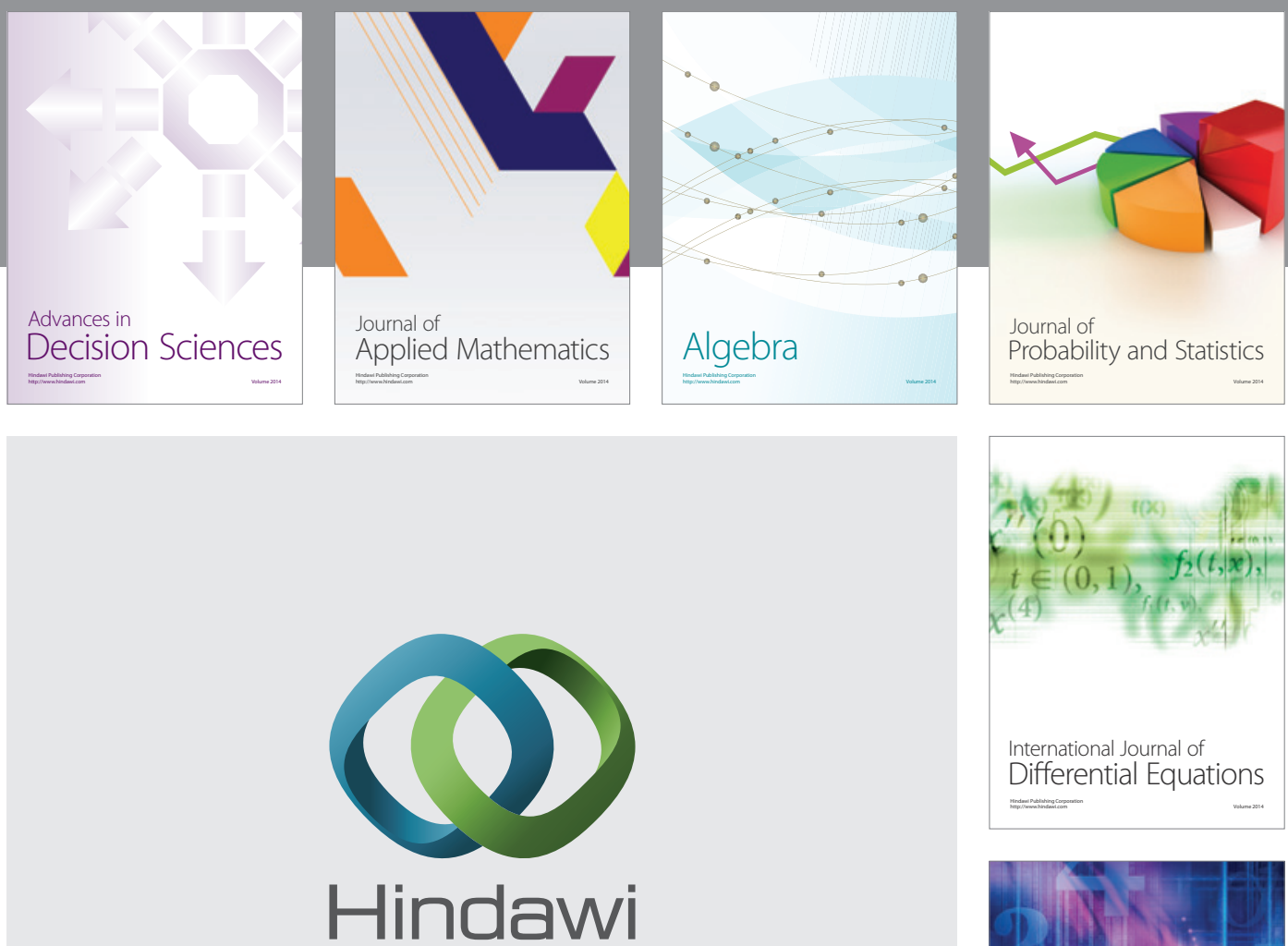

Submit your manuscripts at http://www.hindawi.com
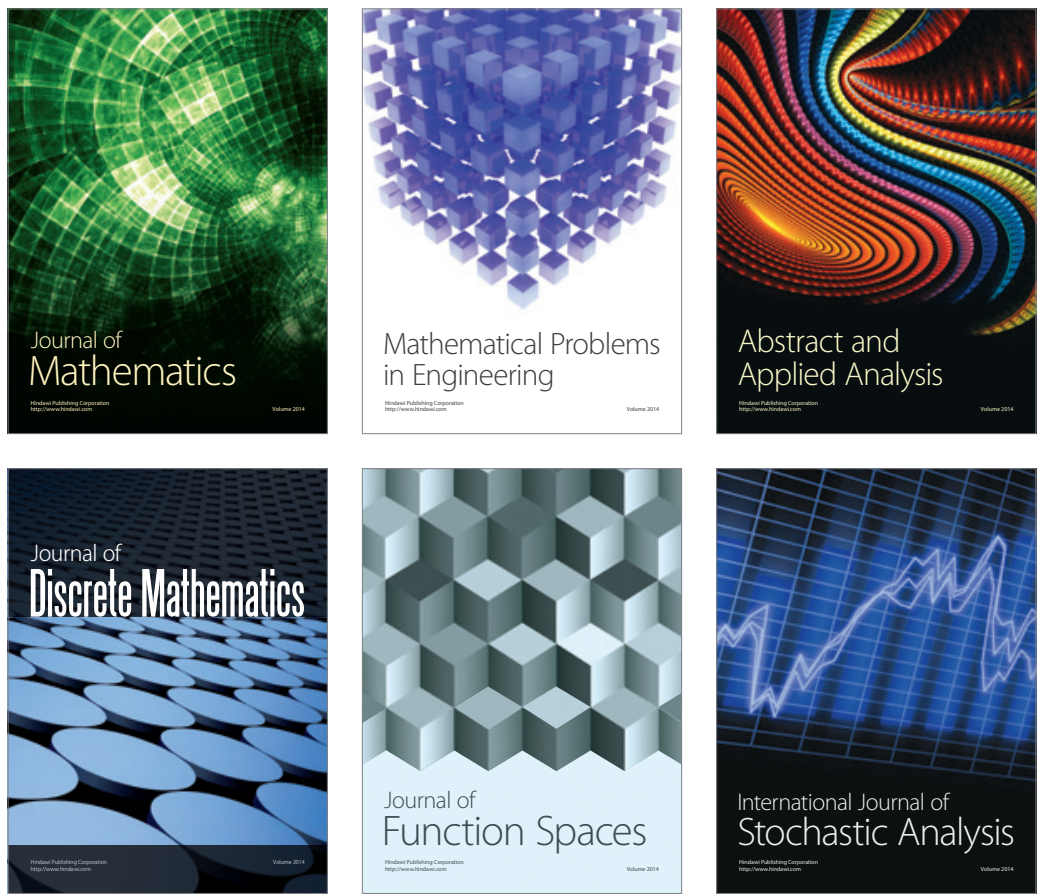

Journal of

Function Spaces

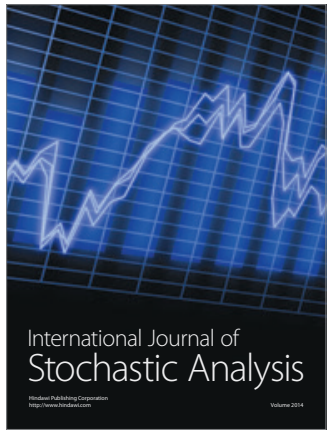

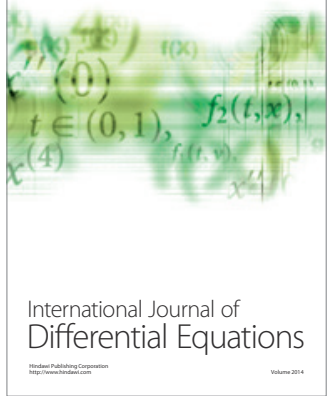
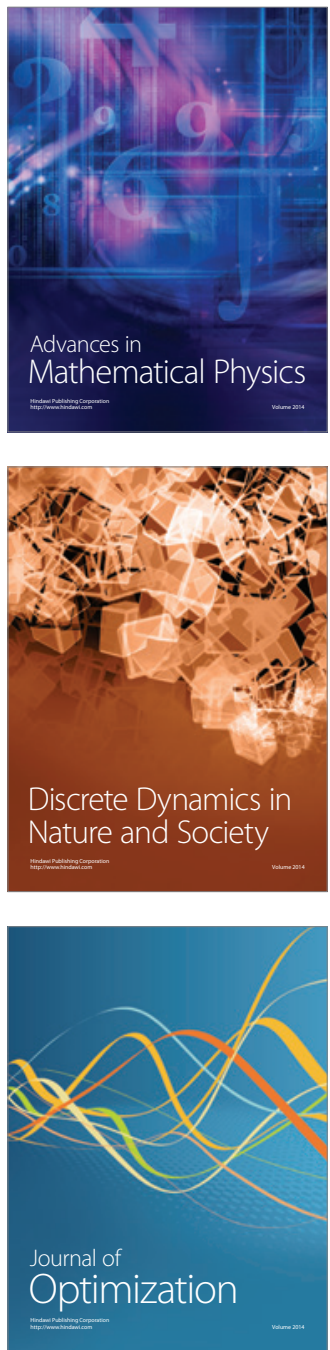